\title{
The ins and outs of on and off
}

\author{
Robert M. Sapolsky, Dora Y. Ho, and John McLaughlin
}

The holy grail for many clinicians is to intervene therapeutically at the level of the genome, either to correct a congenital disorder or to bolster cellular function during an insult. This decade has witnessed the emergence of the nascent discipline of gene therapy with, to date, a disappointingly poor ratio of expectations to actual progress. In this issue, a paper by Harding and colleagues ${ }^{1}$ describes a promising regulatable gene delivery vector for use in the nervous system, a realm where the hopes and accomplishments of gene therapy have been particularly discrepant.

Few diseases compromise the core of who we are like those of the brain. Because of the predominately post-mitotic nature of the nervous system, a neuron lost to injury is a neuron gone forever. What's more, the post-mitotic state of neural cells precludes many of the more successful gene therapy techniques, for the most part limiting neuronal gene therapy to vectors derived from viruses capable of infecting neurons, such as herpes simplex virus 1 or adenovirus (e.g., see ref. 2).

To date, most neuronal gene therapy systems have been constitutive. An inducible system in which expression can be regulated at will with a simple exogenous signal would thus be of great benefit. The tetracycline-responsive promoter approach of Gossen and Bujard ${ }^{3}$ appears to be such a system. It is based on the Tn10 tetracycline-resistance operon of Escherichia coli, where expression of tetracycline resistance genes is repressed by the binding of tetracycline repressor (tet $R$ ) to operator sequences (tetO) located within the promoter region of the tet operon. In the presence of tetracycline, the repressor is inhibited from binding to the operator and transcription is thus derepressed. By fusing tet $R$ with a viral transcriptional transactivation domain (VP16 from herpes simplex virus) and putting the gene of interest under the control of a modified promoter with multiple copies of tet $\mathrm{O}$, transgene expression can be turned on by the TetR-VP16 hybrid (called tTA) in the absence of tetracycline and turned off in its presence (the "Tet-off" system). The same

Robert M. Sapolsky is professor, Dora Y. Ho is research associate, and John McLaughlin is research assistant at the Department of Biological Sciences, Stanford University, Stanford, CA 94305 (sapolsky@leland.stanford.edu). investigators later developed an inverse tetracycline system ${ }^{4}$ employing a new TetR-VP16 hybrid called rtTA in which the presence of tetracycline this time triggers transcription (the "Tet-on" system).

Such vectors are appealing for neurological applications both because they are highly

\section{IMAGE \\ UNAVAILABLE FOR COPYRIGHT REASONS}

Flgure1. Getting it "on" and "off" with the Tet. In the Tet system, a first vector constitutively expresses a transactivator, which acts on the second vector to drive expression of the transgene. In the "off" system (A), activity of the transactivator is blocked by tetracycline, whereas the transactivator requires tetracycline in the "on" system (B).

responsive and because tetracycline analogs readily pass the blood-brain barrier. An intriguing example of how one could apply this technology would be to administer an "On" vector carrying a neuroprotective gene to an individual at risk for stroke and, should an insult occur, activate expression of the gene by infusion of a tetracycline analog.

Such tetracycline-regulated vectors have been used in the rodent brain ${ }^{5,6}$, but have been limited by the interrelated problems of high basal "leakiness" of expression and, thus, relatively low inducibility. Harding and colleagues speculated that the original system suffers from basal leakiness due to "spill-over" of activation from the constitutive promoter to the inducible transgene, which are very close together on the same vector. To get around this, they used a system with two elements, one containing a tetracycline-controlled transactivator (tTA or rtTA) linked to a strong human cytomegalovirus promoter, the other containing the transgene of interest controlled by a minimal promoter containing tetO. In previous approaches, both of these elements were contained within the same vector.
While the paper does not contain any quantitative data (instead, documenting expression of a fluorescent reporter gene with photomicrographs), it appears that this modification greatly reduces basal expression and thus enhances inducibility. Using this "binary" approach, the authors constructed both "On" and "Off" ver-

sions of the system and, in the most impressive feature of the paper, showed that transgene expression could be switched on and off repeatedly over the course of months.

Naturally, this useful advance has some problems. In neurological disease, gene therapy requires the chance event of a vector virion infecting a neuron; the binary approach used here requires the even rarer event of a neuron being infected by both the transactivator and the transgene virions. Adenoviral vectors can be generated at extremely high titers such that, despite a relatively low efficiency of infection, one could utilize the carpetbombing approach needed to get a decent number of cells doubly infected. However, such high adenoviral titers can often cause inflammation and neurotoxicity.

Another issue is how fast expression is initiated or terminated with tetracycline manipulation. The paper gives no information regarding the speed of initiation, but termination appears to take some days with both the "On" and "Off" approaches.

This would preclude the use of any genes which, while neuroprotective immediately following an insult, would be deleterious if sustainedly overexpressed. Finally, most investigators have reported a substantial drop-off in adenoviral expression over time. While the present authors hint at no such decline; this is hard to evaluate with the nonquantitative data. If true, it would represent a substantial advance worth understanding.

In short, right on cue, more work is needed. Neither this nor any other vector delivery systems designed to be neuroprotective are likely to be in the pharmacy soon, tucked between the crates of Viagra. Nonetheless, the field continues to slowly approach actual clinical applications, and the present paper constitutes an important methodological contribution.

\footnotetext{
1. Harding, T. et al. 1998. Nature Biotechnology 16:556-561.

2. Ho, D. and Sapolsky, R. 1997 Sci. Am. July, p. 116

3. Gossen, M. and Bujard, H. 1992. Proc Natl Acad Sci USA 89:5547-5550.

4. Gossen, M, et al. 1995. Science 268:1766-1770.

5. Ho, D., McLaughlin, J., Sapolsky, R. 1996. Mol. Brain Res. 41:200.

6. Fotaki, M., Pink, J., and Mous, J. 1997. Gene Ther. 4:901-908.
} 